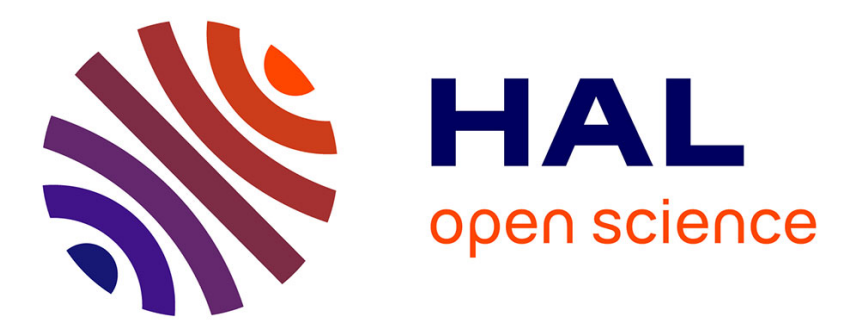

\title{
Magnetic field dependence of the coherence length and penetration depth of MgB2 single crystals
}

Thierry Klein, L. Lyard, J. Marcus, Z. Holanova, C. Marcenat

\section{To cite this version:}

Thierry Klein, L. Lyard, J. Marcus, Z. Holanova, C. Marcenat. Magnetic field dependence of the coherence length and penetration depth of MgB2 single crystals. Physical Review B: Condensed Matter and Materials Physics (1998-2015), 2006, 73, pp.184513. 10.1103/PhysRevB.73.184513 . hal00957192

\section{HAL Id: hal-00957192 \\ https://hal.science/hal-00957192}

Submitted on 10 Mar 2014

HAL is a multi-disciplinary open access archive for the deposit and dissemination of scientific research documents, whether they are published or not. The documents may come from teaching and research institutions in France or abroad, or from public or private research centers.
L'archive ouverte pluridisciplinaire HAL, est destinée au dépôt et à la diffusion de documents scientifiques de niveau recherche, publiés ou non, émanant des établissements d'enseignement et de recherche français ou étrangers, des laboratoires publics ou privés. 


\title{
Magnetic field dependence of the coherence length and penetration depth of $\mathrm{MgB}_{2}$ single crystals
}

\author{
T. Klein, ${ }^{1,2}$ L. Lyard, ${ }^{1}$ J. Marcus, ${ }^{1}$ Z. Holanova,,${ }^{1}$ and C. Marcenat ${ }^{3}$ \\ ${ }^{1}$ Laboratoire d'Etudes des Propriétés Electroniques des Solides, CNRS, B.P. 166, 38042 Grenoble Cedex 9, France \\ ${ }^{2}$ Institut Universitaire de France and Université Joseph Fourier, B.P. 53, 38041 Grenoble Cedex 9, France \\ ${ }^{3}$ Département de Recherche Fondamental sur la Matiére Condensée, CEA-Grenoble, F-38054 Grenoble Cedex 9, France
}

(Received 6 August 2005; revised manuscript received 6 April 2006; published 11 May 2006)

\begin{abstract}
We report on specific heat and Hall probe magnetization measurements in magnesium diboride single crystals. A magnetic field dependence of the coherence length $(\xi)$ has been deduced from the former assuming that the electronic excitations are localized in field dependent vortex cores in which case $\xi$ is related to the Sommerfeld coefficient $\gamma=\Delta C_{p} /\left.T\right|_{T \rightarrow 0}$ throughout, $\gamma \propto\left[\xi(H) / a_{0}\right]^{2}$ ( $a_{0}$ being the vortex spacing). The reversible part of the magnetization has been analyzed with a phenomenological Ginzburg-Landau model introducing field dependent parameters (i.e., penetration depth $\lambda$ and $\xi$ ) which account for the decreasing contribution of the $\pi$-band with increasing field. This approach perfectly reproduces the experimental data by combining the field dependence of $\xi$ deduced from $C_{p}\left(1 / \xi^{2} \sim \sqrt{B}\right)$ with an almost linear increase of $\lambda$ from $\sim 450 \AA$ at low field to $\sim 700 \AA$ close to $H_{c 2}$. These field dependences can then be used to consistently describe the field dependence of the critical current density, small angle neutron scattering form factor, and muon spin relaxation rate.

DOI: 10.1103/PhysRevB.73.184513

PACS number(s): 74.70.Ad, 74.25.Ha, 74.25.Op, 74.25.Qt
\end{abstract}

\section{INTRODUCTION}

One of the most salient consequences of the coexistence of two superconducting gaps in magnesium diboride $\left(\mathrm{MgB}_{2}\right)$ is the anomalous temperature dependence of the anisotropy of the upper critical field, ${ }^{1} \Gamma_{H_{c 2}}=H_{c 2}^{a b} / H_{c 2}^{c}\left(H_{c 2}^{a b}\right.$ and $H_{c 2}^{c}$ being the upper critical fields parallel to the $a b$-planes and $c$-axis, respectively). On the other hand, the lower critical field $\left(H_{c 1}\right)$ is almost isotropic at low temperature ${ }^{2}$ suggesting that the anisotropy parameter $\Gamma=\xi_{a b} / \xi_{c}=\lambda_{c} / \lambda_{a b}\left(\lambda_{i}\right.$ and $\xi_{i}$ being the penetration depth and the coherence length in the related direction, respectively) is also field dependent rising from $\approx \Gamma_{H_{c 1}} \sim 1$ at low field to $\Gamma_{H_{c 2}} \sim 5-6$ at high field. Indeed, as suggested for instance by point contact spectroscopy ${ }^{3}$ and small angle neutron scattering (SANS) ${ }^{4}$ experiments, the socalled $\pi$-band is rapidly suppressed by magnetic field and the anisotropy is then mainly given by the parameters of the quasi-2D $\sigma$-band above some "crossover" field on the order of 0.5-1 T. On the other hand, at low field, the anisotropy must be averaged over the entire Fermi surface ${ }^{7}$ leading to $\Gamma \sim 1$ (at low temperature) in good agreement with $H_{c 1}$ measurements. ${ }^{2}$

Furthermore, a kink is clearly visible in the field dependence of the Sommerfeld coefficient $\gamma=\Delta C_{p} /\left.T\right|_{T \rightarrow 0}\left(\Delta C_{p}\right.$ being the field dependent electronic contribution to the specific heat). ${ }^{8}$ The rapid increase of $\gamma$ at low field, reaching $\approx 50 \%$ of the normal state value $\left(\gamma_{N}\right)$ at $0.5 \mathrm{~T}$ has again been attributed to a rapid filling of the $\pi$-band with increasing magnetic field. ${ }^{8}$ Finally, the form factor in small angle neutron scattering (SANS) measurements, ${ }^{4}$ the muon spin relaxation rate ${ }^{9}$ and the logarithmic derivative of the reversible magnetization $^{2,9}\left[d M_{\text {rev }} / d \operatorname{Ln}(H)\right]$ which are all expected to be proportional to the superfluid density $\left(n_{s} \propto 1 / \lambda^{2}\right)$ present an anomalous field dependence in $\mathrm{MgB}_{2}$.

We show here that the anomalous field dependences of the specific heat, reversible magnetization, critical current density, SANS form factor and muon spin relaxation rate can all be consistently described by assuming that the penetration depth and the coherence length are field dependent. The field dependence of $\xi$ has been deduced from $C_{p}$ assuming that electronic excitations are localized in field dependent vortex cores such as $\Delta C_{p} /\left.T\right|_{T \rightarrow 0} \propto\left[\xi(H) / a_{0}\right]^{2}$ (where $a_{0}$ is the vortex spacing) and the reversible magnetization has been analyzed using a phenomenological Ginzburg-Landau approach introducing field dependent $\xi$ and $\lambda$ values. Indeed, as pointed out recently by Eisterer et al., ${ }^{5} \mathrm{MgB}_{2}$ can be described by a one-gap Ginzburg-Landau model assuming that the order parameters of the two bands are related together by a field dependent parameter which accounts for the field dependence of the relative contributions of the two bands. This progressive change of the contributions of the two bands shows up as field dependent $\xi$ and $\lambda$ values. We will only report on the field dependence of $\xi_{a b}$ and $\lambda_{a b}$ (i.e., deduced from measurements performed with $H \|_{c}$ ) but $\xi_{c}$ and $\lambda_{c}$ are also expected to be field dependent consistently with the field dependence of the anisotropy parameter. In the previously published analysis, ${ }^{2,4,9}$ a field dependence of the coherence length was not taken into account leading to a large overestimation of $\lambda$.

\section{EXPERIMENT}

Specific heat measurements have been performed on a small $\mathrm{MgB}_{2}$ single crystal using an ac technique as described elsewhere. ${ }^{1}$ This crystal has been grown by annealing $\mathrm{Mg}$ and $\mathrm{B}$ in stoechiometric composition at $1150{ }^{\circ} \mathrm{C}$ for $70 \mathrm{~h}$ in a closed iron container. This high sensitivity ac technique is not only very well adapted to measure the specific heat of very small samples (a few $\mu \mathrm{g}$ in our case) but also to carry continuous measurements during field sweeps at a given temperature (in Ref. 8, $\gamma$ was deduced from the temperature dependence of $C_{p}$ for a limited number of magnetic fields). We were thus able to obtain the field dependence of the Sommerfeld coefficient continuously on the entire field range 


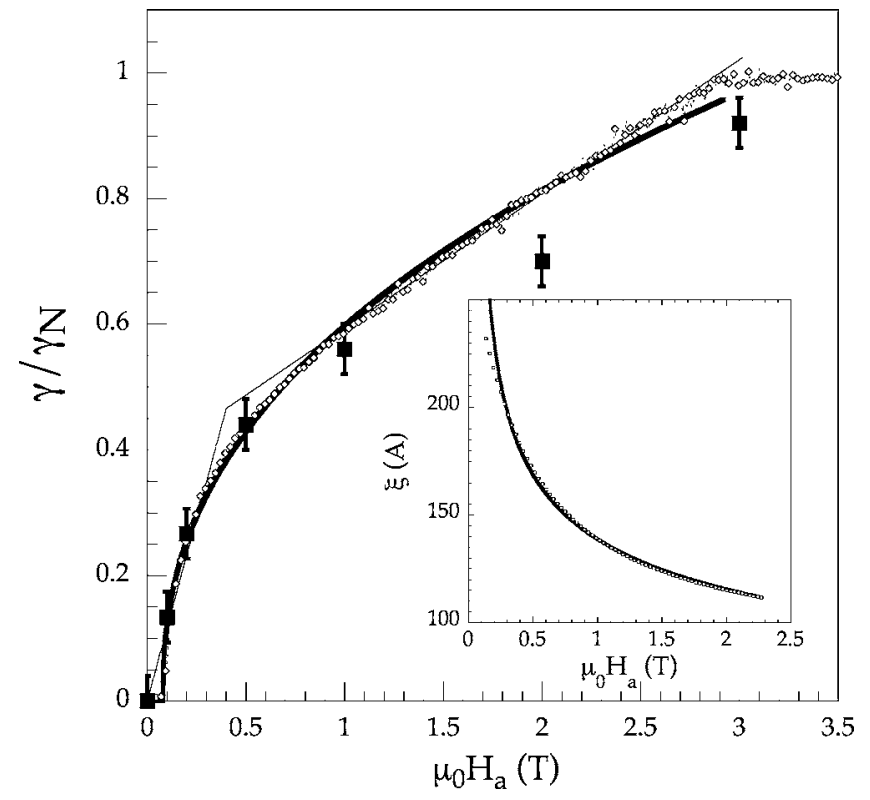

FIG. 1. Magnetic field dependence of the Sommerfeld coefficient $\gamma$ at $T=2.5 \mathrm{~K}$ (open diamonds). The bold line is a $\sqrt{H-H_{p}}$ dependence $\left(H_{p}\right.$ being the first penetration field) and the two straight lines would correspond to independent bands closing at two different fields (see text for details), closed squares, data from Ref. 6 , note that the two samples have slightly different $H_{c 2}$ values. Inset, magnetic field dependence of the coherence length (open squares) $\xi \propto \sqrt{C_{p} / H} \propto 1 / H^{0.25}$ (solid line).

from $H_{c 1}$ to $H_{c 2}$ (at $2.5 \mathrm{~K}$, see Fig. 1). A precise in-situ calibration of the thermocouple used to record the temperature oscillations was obtained from measurements on ultrapure silicon.

Local magnetization measurements were performed on the same single crystal using a miniature Hall probe array. The field distribution in the sample has thus been recorded from various applied fields $H \| c$ at $T=4.2 \mathrm{~K}$. As shown in Ref. 6 , for low $\mu_{0} H_{a}$ values (typically $\leq 0.15 \mathrm{~T}$ ) the field distribution presents a "dome shape" characteristic of the presence of strong surface barriers. The effect of those barriers decreases rapidly with field and the irreversibility is again dominated by bulk pinning effects at high fields. The reversible part $\left(M_{\mathrm{rev}}^{\text {loc }}\right)$ of the "magnetization" $\left(B-\mu_{0} H_{a}\right.$, where $B$ is the induction at the center of the sample) has thus been defined as the average between the increasing and decreasing branches of the "magnetization loop" (dotted lines in Fig. 2). Obviously, such a procedure is only correct for "high" magnetic fields (i.e., above $\sim 0.2 \mathrm{~T}$ ) and it is important to note that the irreversible part of the magnetization decreases very rapidly with field. The reversible contribution is thus very well defined above $\sim 0.2-0.3 \mathrm{~T}$. The critical current density has then been defined as the width of the magnetization loop $\left(\Delta B / 2 \mu_{0} w\right.$, where $2 w$ is the width of the sample).

\section{RESULTS AND DISCUSSION}

\section{A. Specific heat measurements: field dependence of the coherence length}

Figure 1 displays the magnetic field dependence of the Sommerfeld coefficient $\gamma$ at $T=2.5 \mathrm{~K}$. It is important to note

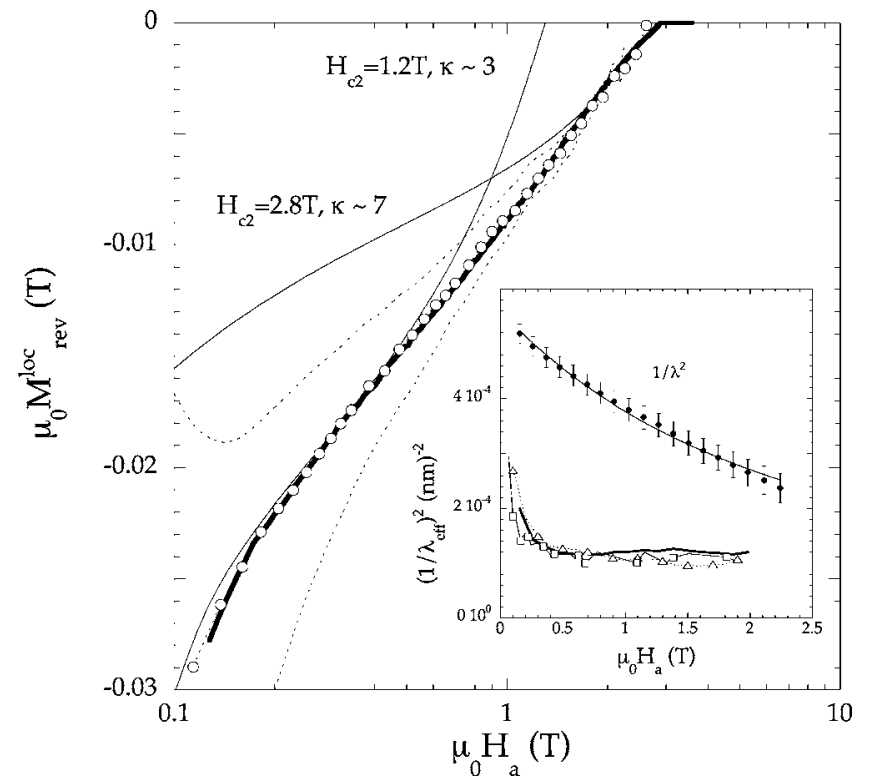

FIG. 2. Magnetic field dependence of the reversible part of the local magnetization $B-\mu_{0} H_{a}$ (open circles). The dotted lines are the local magnetization branches for ascending and descending fields. The thick line is the calculated curve with $\xi(H)$ given by the specific heat and a linear $\lambda(H)$. The thin lines are calculations for conventional materials with the indicated $\kappa$ and $H_{c 2}$ values. Inset, magnetic field dependence of $1 / \lambda^{2}$ (see text for details) compared to $1 / \lambda_{\mathrm{eff}}^{2} \propto d M_{\text {rev }} / d \operatorname{Ln}(H)$, calculated (thick line), from SQUID data (Ref. 7) (triangles) and from Hall probe magnetometry (Ref. 2) (squares). The solid line in the $1 / \lambda^{2}$ data corresponds to the linear field dependence of $\lambda$.

that we restricted ourself in the following analysis to fields larger than $\sim 0.15 \mathrm{~T}$ for which the applied field is close to the induction $B$. Indeed, for fields up to a few $\mu_{0} H_{p}$ $\sim 0.05 \mathrm{~T}$, the first penetration field, the measurements are strongly hysteretic reflecting different vortex distributions in the sample. ${ }^{6}$

As discussed in Ref. 8 , in $\mathrm{MgB}_{2}$ the nonlinear field dependence of the Sommerfeld coefficient (see Fig. 1) could be qualitatively understood by considering the contributions of the two bands as independent and thus writing that $\gamma=\omega \gamma_{\pi}$ $+(1-\omega) \gamma_{\sigma}$ where $\gamma_{\pi}$ and $\gamma_{\sigma}$ are the contributions of the two bands and $\omega$ the weight of the $\pi$ band (on the order of 1/2). Assuming that the electronic excitations are localized in the vortex cores of volume $\sim \xi^{2} t$ (where $t$ is the thickness of the sample) one gets $\gamma_{i} \propto\left(n_{V} \gamma_{N}\right) \times\left(\xi_{i}^{2} t / S t\right)$ where $i=\pi$ or $\sigma, S$ is the sample surface and $n_{V}$ the number of vortices. As $n_{V} / S$ $\propto 1 / a_{0}^{2}$ ( $a_{0}$ being the vortex spacing), one finally obtains $\gamma_{i}$ $\propto\left(\xi_{i} / a_{0}\right)^{2}$ for $H<H_{i}=\Phi_{0} / 2 \pi \mu_{0} \xi_{i}^{2} . \gamma_{i}=\gamma_{N}$ for $H>H_{i}$ and $\gamma$ is thus expected to present two linear behaviors (for $H$ $<H_{\pi}$ and $H>H_{\pi}$, respectively) as shown in Fig. 1 (solid lines with $\omega=0.4$ and $\mu_{0} H_{\pi}=0.5 \mathrm{~T}$ ). Note however, that the proximity of $H_{p}$ may cast some doubt on the linear field dependence of $\gamma$ observed by the authors of Ref. 8 in the low field range.

The magnetic field dependence of the Sommerfeld coefficient at $T=2.5 \mathrm{~K}$ is displayed in the main panel of Fig. 1 for $H \| c$. The starting point of our approach is to consider that, due to the coupling of the two bands, the system can be 
described by only one, but field dependent, $\xi$ value. Still assuming that the electronic excitations are localized in the vortex cores, one hence has

$$
\gamma \propto\left(\frac{\xi(H)}{a_{0}}\right)^{2} .
$$

One then directly gets $\xi \propto \sqrt{\gamma / H}$ with $\xi\left(H=H_{c 2}\right)$ $=\sqrt{\Phi_{0} / 2 \pi \mu_{0} H_{c 2}}$ (see inset of Fig. 1). This field dependence is indeed reflecting the decrease of the contribution of the $\pi$-band and our approach is, in this sense, equivalent to the one proposed by Bouquet et al. ${ }^{8}$ but introducing here an "effective" coherence length which takes into account the decrease of the contribution of the $\pi$-band through a smooth variation between $H_{p}$ and $H_{c 2}$ with $1 / \xi^{2} \propto \sqrt{H-H_{p}} \approx \sqrt{B}$. It is tempting to associate the $\xi(H \rightarrow 0)$ value to the $\pi$-band using the standard BCS expression $\xi_{\pi} \sim \hbar v_{F} / \pi \Delta_{\pi} \sim 500 \AA$ (where $v_{F}$ and $\Delta_{\pi}$ are the Fermi velocity and gap value, respectively). This value is in agreement with tunneling spectroscopy measurements ${ }^{18}$ but, as discussed by Zhitomirsky and Dao, ${ }^{19}$ the size of the vortex core at low field is actually not expected to be given by the BCS expression but $\xi(H \rightarrow 0)$ is expected to be on the order to $1-2 \times \xi_{\sigma}$ for realistic parameters ${ }^{19}$ in agreement with our data which suggest that $\xi(H \sim 0.2 T) \sim 2-2.5 \times \xi_{\sigma}$.

Note that, similar nonlinearities have been observed in many other superconductors (from $\mathrm{NbSe}_{2}$ to borocarbides, ${ }^{10}$ bismuthates ${ }^{11}$ or cuprates ${ }^{12}$ ) and a shrinking of the vortex cores for increasing fields has actually been recently predicted by Kogan and Zhelezina ${ }^{13}$ for classical one gap superconductors in the clean limit. The predicted behavior is similar to the one obtained here for $H \|_{c}$ but a much steeper variation has been reported for $H \| a b$ by Bouquet et al. ${ }^{8}(\gamma$ reaching $50 \%$ of its normal state value for $H / H_{c 2} \sim 1 / 20$ ) clearly suggesting an influence of the two-gap nature in $\mathrm{MgB}_{2}$. A similar shrinkage of the vortex core (and corresponding increase of the penetration depth, see below) has also been reported recently by Callaghan et al. ${ }^{14}$ in $\mathrm{NbSe}_{2}$ (from $\mu \mathrm{SR}$ measurements). Even though this system has been thought of for a long time as being a one gap $s$-wave superconductor, there is now convincing evidence for the existence of multiple gaps. ${ }^{15,16}$ As previously pointed out by Boaknin et al. ${ }^{16}$ thermal conductivity measurements can be scaled tothe classical $s$-wave behavior introducing a low field delocalization length $\xi(H \rightarrow 0)$ on the order of $\sim 3 \times \xi$ (i.e., corresponding to an effective upper critical field on the order of $H_{c 2} / 9$ ). A similar increase of the thermal conductivity at low field has also been observed in $\mathrm{MgB}_{2}$ (Refs. 16 and 17 and such a "scaling" is consistent with our description in which $\xi(H \rightarrow 0) \gg \xi\left(H=H_{c 2}\right)$ (i.e., the "low field upper critical field" is much smaller than $H_{c 2}$ ). We will show below that it is of fundamental importance to include this field dependence of the coherence length in the analysis of other physical quantities such as the magnetization, SANS form factor and muon polarization rate.

\section{B. Magnetization measurements: field dependence of the penetration depth}

The local induction at the center of the sample has been measured using a miniature Hall probe for increasing and decreasing magnetic field (at $T=4.2 \mathrm{~K}$ see dotted lines in Fig. 2). As shown in Ref. 6, at low field the irreversibility is dominated by geometrical barriers which renders the determination of the reversible part of the magnetization difficult. However, for $H$ larger than a few $\mu_{0} H_{p} \sim 0.05 \mathrm{~T}$, the irreversibility related to those barriers vanishes and the remaining irreversibility can thus be attributed to the presence of a small critical current due to bulk pinning. The reversible part $\left(M_{\mathrm{rev}}^{\mathrm{loc}}\right)$ of the "local magnetization" $\mu_{0} M^{\mathrm{loc}}=B-\mu_{0} H_{a}$ has thus been defined as the average between the ascending and descending branches of the magnetization loop.

In type II superconductors, the reversible magnetization $\left(M_{\text {rev }}\right)$ is entirely defined by $h=H / H_{c 2}$ and $\kappa=\lambda / \xi,{ }^{21}$

$$
M_{\text {rev }} / H_{c 2}=-\frac{1-h}{\left(2 \kappa^{2}-1\right) \beta_{A}+1}
$$

at high field (Abrikosov regime, where $\beta_{A}$ is the Abrikosov coefficient) whereas in the intermediate field range (London regime) $M_{\text {rev }}$ is expected to vary linearly with $\ln H$ as

$$
M_{\text {rev }} / H_{c 2}=-\frac{1}{4 \kappa^{2}} \ln \left(\frac{0.358}{h}\right) .
$$

Finally, $M_{\text {rev }}$ is expected to tend towards $-H_{c 1}$ for $B \rightarrow 0$ with a vertical slope. Surprisingly, in magnesium diborides, the linear variation of $M$ expected for $H \geq H_{c 2} / 3$ is not observed and an almost logarithmic regime is observed all the way up to $H_{c 2}$ (see Fig. 2). A similar behavior has also been reported by several groups from global measurements and is thus not related to our local measurements. ${ }^{2,9}$ Some discrepancy may be expected due to the limitations of the Ginzburg-Landau (GL) model ${ }^{20}$ but in the case of $\mathrm{MgB}_{2}$ this approach does not work at all. As pointed out by Zehetmayer et al. ${ }^{22}$ the single band GL model can however still be used to fit the reversible magnetization in the low and high field regimes but only by using very different parameters (i.e., coherence length and penetration depth). It is thus only possible to fit the data on a very limited field range using a unique set of $\left(H_{c 2}, \kappa\right)$ values.

Introducing the field dependence of $\xi$ deduced from our specific heat measurements in some effective "upper critical field" $\left[\mu_{0} H_{c 2}^{*}=\Phi_{0} / 2 \pi \xi(H)^{2}\right]$ we have adjusted the reversible magnetization deduced from the GL model to our experimental data in the entire field range with only one free parameter, $\lambda=\kappa \times \xi$. $M_{\text {rev }}$ has been calculated from the interpolation formula calculated by Brandt, ${ }^{21}$

$$
M_{\text {rev }}=-\frac{H_{c 2}}{4 \kappa^{2}} \ln \left(1+\frac{1-h}{h}\left(0.357+2.89 h-1.581 h^{2}\right)\right)
$$

taking $h=H / H_{c 2}^{*}$. As pointed out above, in this phenomenological approach, $H_{c 2}^{*}$ does not correspond anymore to the "true" upper critical field (on the order of $2.9 \mathrm{~T}$ along the $c$ direction) but to a field dependent parameter containing the field dependence of the coherence length. Taking $\xi_{a b}$ $\propto 1 / H^{0.25}$, an almost linear field dependence for $\lambda_{a b}$ reproduces very well the "pseudologarithmic" behavior observed experimentally. The corresponding $M_{\mathrm{rev}}^{\text {loc }}$ curve reported in Fig. 2 (bold line) has been calculated assimilating our sample 
to an ellipsoid with a demagnetization factor $N \approx 0.6$ [and thus writing $M_{\text {rev }}^{\text {loc }}=(1-N) M_{\text {rev }}$. Such a demagnetization factor leads to the correct $H_{p}$ value [taking $H_{c 1}=0.11 \mathrm{~T}$ (Ref. 6)] but has indeed only little physical meaning at low field as we demonstrated that "corner effects" play a dominant role in $\mathrm{MgB}_{2}$ (Ref. 6) (we thus restricted our analysis for $\mu_{0} H$ $\geq 0.2 \mathrm{~T}$ ). The magnetization jump associated to those edge currents has thus not been taken explicitly into account but for $H$ larger than a few $H_{p}$ geometrical corrections rapidly become negligible $\left(B \rightarrow \mu_{0} H_{a}\right)$. The use of a demagnetization coefficient is consistent with the fact that our field profiles are becoming very flat above $\sim 0.2 \mathrm{~T}$ (i.e., the irreversible contribution is very small) and the magnetization is thus almost homogeneous in this field range. This approximation is further validated by the fact that our local measurements only differ from the global measurements (see for instance Ref. 9 for SQUID measurements) by a scaling factor for $H_{a} \gg H_{p}$ [here equal to $\left.\sim(1-N)\right]$. The choice of $N$ does not influence the determination of $\lambda_{a b}(H)$. Taking, for instance $N=0.7$ (which would correspond to an ellipsoid of similar thickness/width ratio but with a slightly lower $H_{p}$ value) leads to $\lambda$ values about $10 \%$ smaller than the former ones but presenting a very similar field dependence.

As for $\xi$, we obtain a continuous and smooth variation for $\lambda$ between $H_{p}$ and $H_{c 2}$ rather than a crossover between a low $\left(\mu_{0} H_{c 2}=1.2 \mathrm{~T}, \kappa=3\right)$ and a high $\left(\mu_{0} H_{c 2}=2.8 \mathrm{~T}, \kappa=7\right)$ field regime. We obtained $\lambda(H \rightarrow 0) \sim 450 \AA$ and $\lambda\left(H \rightarrow H_{c 2}\right)$ $\sim 750 \AA$. These values are in good agreement with those obtained by Zehetmayer et al. $22 . \sim 510 \AA$ and $760 \AA$ at low and high field, respectively (and correspondingly $\xi$ varying from $\sim 174 \AA$ to $104 \AA$ ). The low field $\lambda_{a b}$ values is also in good agreement with the theoretical one calculated by Golubov et al. ${ }^{23}$ in the clean limit $(\sim 400 \AA)$. In the clean limit "assumption" $\lambda$ is expected to be almost isotropic which is in good agreement with the almost isotropic $H_{c 1}$ previously observed in Ref. 2 as well as with SANS measurements (see Ref. 24 and reference therein). The increase of $\lambda_{a b}$ with increasing field is indeed reflecting the decrease of the superfluid density as superconductivity in the $\pi$-band is progressively destroyed by $H$. A $\sim 50 \%$ increase is hence consistent with a decrease of the superfluid density by a factor of 2 (corresponding to $\omega=0.5$ ). Note that $\kappa$ is ranging from $\sim 2-3$ at low field to $\sim 7$ close to $H_{c 2}$ and $\mathrm{MgB}_{2}$ is thus close to a "type I" superconductor in the low field limit.

The field dependence of $1 / \lambda_{\text {eff }}=\left(8 \pi \mu_{0} / \Phi_{0}\right)$ $\times d M_{\text {rev }} / d \ln (H)$ is displayed in the inset of Fig. 2 together with experimental data deduced from SQUID measurements by Angst $e t a l .{ }^{9}$ as well as with the values that we previously obtained on another sample. ${ }^{2}$ In "classical" systems, $d M_{\text {rev }} / d \ln (H)$ is directly proportional to $1 / \lambda^{2}$ (i.e., $\lambda=\lambda_{\text {eff }}$ ), but as shown in the inset of Fig. 2, this is not the case in $\mathrm{MgB}_{2}$. Indeed, an almost field independent (above $0.5 \mathrm{~T}$ ) $\lambda_{\text {eff }}$ value on the order of $900 \AA$ is deduced from this derivative whereas $\lambda_{a b}$ actually increases from $\sim 450$ to $\sim 700 \AA$. Note that a $\lambda_{a b}$ value on the order of $900 \AA$ would suggest that the system is in the dirty limit which would be inconsistent with the isotropy of $H_{c 1}$ (indeed, $\lambda$ and hence $H_{c 1}$ are expected to present an anisotropy on the order of 3 in the dirty limit ${ }^{23}$ ). Neglecting the field dependence of $\xi$ and deducing $\lambda$ from the logarithmic derivative of the reversible magnetization thus leads to a large overestimation of $\lambda$.

A phenomenological approach very similar to ours has been proposed very recently by Eisterer et $a l^{5}$ The authors suggested that the two band free energy functional ${ }^{19}$ $\left(F_{\mathrm{GL}}^{\mathrm{twoband}}\right)$ can be reduced to an effective one band functional $\left(F_{\mathrm{GL}}\right)$ assuming that $\psi_{\pi}=\rho \times \psi_{\sigma}$ where $\rho$ is a field dependent parameter accounting for the progressive decrease of the contribution of the $\pi$-band to the superfluid density $\left(\psi_{\pi}\right.$ and $\psi_{\sigma}$ being the order parameters in the $\pi$ and $\sigma$ bands, respectively),

$$
F_{\mathrm{GL}}^{\mathrm{twoband}}\left(E_{c, \pi}, E_{c, \sigma}, E_{\gamma}, \lambda_{\pi}, \lambda_{\sigma}\right)=F_{\mathrm{GL}}\left(E_{c}, \lambda\right),
$$

where $E_{c, \pi}, E_{c, \sigma}, E_{\gamma}, \lambda_{\pi}, \lambda_{\sigma}$ are the condensation energy of the $\pi$ and $\sigma$ bands, the (Josephson type) coupling energy between the two bands and the penetration depth of the corresponding bands, respectively. $E_{c}(\rho)$ and $\lambda(\rho)$ are then some "effective one band" field dependent condensation energy and penetration depth, respectively. However, those quantities are expected to be related to the microscopic parameters of the two bands through ${ }^{5} E_{c}=\left(E_{c, \sigma}+\rho^{2} E_{c, \pi}+\rho E_{\gamma}\right)\left|\Phi_{0, \sigma}\right|^{2}$ and $\lambda=\lambda_{\sigma} \lambda_{\pi} / \sqrt{\lambda_{\pi}^{2}+\rho^{2} \lambda_{\sigma}^{2}\left|\Phi_{0, \sigma}\right|^{2}}$ with $\left|\Phi_{0, \sigma}\right|^{2}=\left(E_{c, \sigma}+\rho^{2} E_{c, \pi}\right.$ $\left.+\rho E_{\gamma}\right) /\left(E_{c, \sigma}-\rho^{4}\left|E_{c, \pi}\right|\right)$. The temperature dependence of $E_{c, \pi}, E_{c, \sigma}, \lambda_{\pi}$, and $\lambda_{\sigma}$ as well as the field dependence of $\lambda$ have then been obtained by minimization of the total Gibbs energy at each field. Note that the authors of Ref. 5 had to use this minimization procedure in order to extract $\lambda(H)$ from their magnetization data as $M_{\text {rev }}$ depends on both $\lambda$ and $\xi$. Our determination is thus more straightforward as we combined specific heat (which depends on $\xi$ only) and magnetization measurements on the same crystal. No field dependence of $\xi$ is presented in Ref. 5 but the $\lambda_{a b}$ values are very similar to those obtained in the present work even though a small kink (not present in our data) was obtained around $\sim 1 \mathrm{~T}$ at low temperature.

Above $\sim 0.5 \mathrm{~T}$ we obtain an almost field independent $E_{c}$ value on the order of $30 \mathrm{~kJ} \mathrm{~m}^{-3}$ in agreement with the values obtained by Eisterer et al. ${ }^{5}$ Indeed, since $E_{c, \pi}$ and $E_{\gamma}$ are much smaller than $E_{c, \sigma}$ and $\rho \rightarrow 0, E_{c}$ rapidly tends towards $E_{c, \sigma}$ and is hence expected to be almost field dependent at high field. However, it is important to note that the $\psi_{\pi}=\rho$ $\times \psi_{\sigma}$ ansatz assumes that the size of the vortex core is almost field independent and might thus not be valid at low field. The microscopic signification of the low field $\xi$ and $\lambda$ parameters thus obviously require further theoretical and experimental work but we will show below that those parameter can be used to consistently describe the field dependence of other physical quantities without introducing any new parameter.

First, field dependent $\lambda_{a b}(H)$ and $\xi_{a b}(H)$ values also influence the field dependence of the critical current density $\left(J_{c}\right)$. Indeed, assuming that vortices are collectively pinned by a large number of weak pins (so-called collective pinning regime), $J_{c}$ is expected to be given by ${ }^{28,29}$

$$
J_{c} \propto \frac{W^{2}}{B r_{p}^{3} c_{44} c_{66}^{2}},
$$

where $W$ is the average pinning strength, $r_{p}$ is the interaction range of the pinning centers and $c_{44}$ and $c_{66}$ are the tilt and 


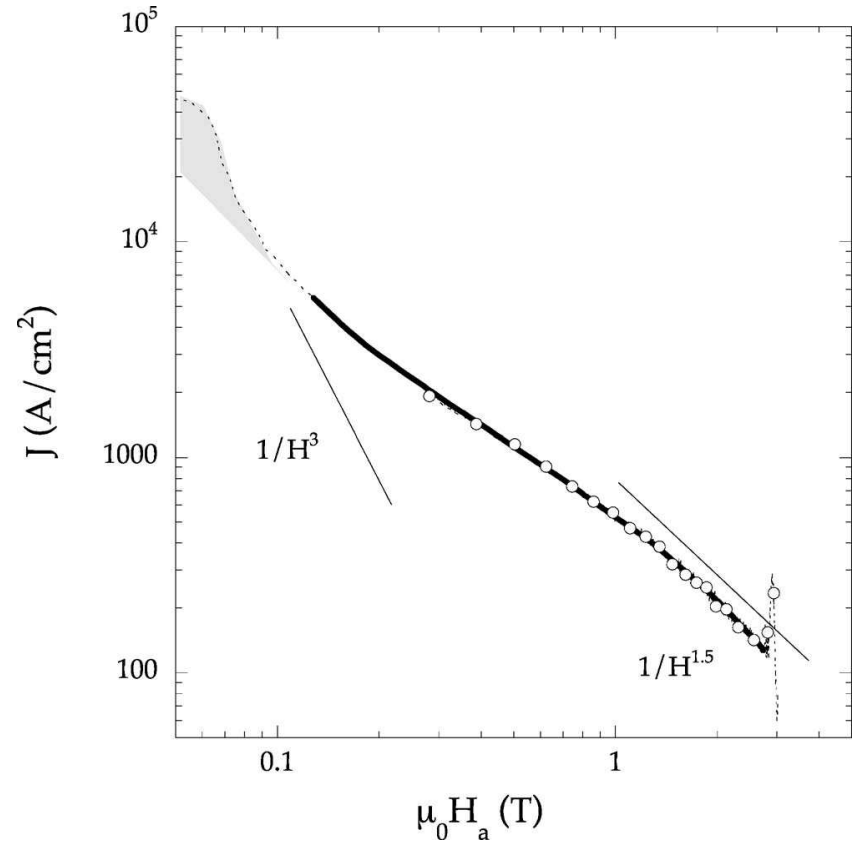

FIG. 3. Magnetic field dependence of the critical current (diamonds) compared to the calculated values (thick line) assuming that large vortex bundles are collectively pinned by a large number of weak pins. The straight lines correspond to the low and large $H$ limits neglecting the field dependence of $\xi$ and $\lambda$ (see text for details). The shaded area at low field schematically represents the increase of the irreversibility due to the presence of geometrical barriers.

shear moduli of the vortex lattice, respectively. Neglecting nonlocal effect in $c_{44}$ (i.e., so-called large bundle regime), one expects at low field ${ }^{28,29}$ (i.e., $\left.h \leq 0.3\right) W \propto h, r_{p} \sim \xi, c_{44}$ $\propto H^{2}$ and $c_{66} \propto H / \lambda^{2}$ which leads to $J_{c} \propto 1 / H^{3}$ for field independent $\xi$ and $\lambda$ values. Similarly, close to $H_{c 2},{ }^{28} W \propto 1-h$, $r_{p} \propto a_{0} \propto 1 / \sqrt{H}, c_{44} \propto H^{2}$, and $c_{66} \propto(1-h) / \lambda^{2}$ and hence $J_{c}$ $\propto 1 / H^{1.5}$.

Figure 3 displays the field dependence of the critical current deduced from the width of the magnetization loop for $\mu_{0} H_{a}<1 \mathrm{~T}$ (neglecting possible creep effects). Note that close to $H_{p}$ the width of the cycle rapidly increases due to the presence of geometrical barriers ${ }^{6}$ and does not correspond anymore to a critical current. At high field, $J_{c}$ is getting very small and could hardly be deduced from our magnetization measurements. The $J_{c}(H)$ curve has thus been deduced from ac-transmittivity measurements following. ${ }^{30}$ Note the presence of a sharp peak effect close to $H_{c 2}$ which will not be discussed here. As shown, at high field, the $J_{c}(H)$ dependence is close to the $1 / H^{1.5}$ law predicted by the collective pinning theory. However as $H$ decreases $J_{c}(H)$ rapidly deviates from the "classical" (i.e., assuming constant $\xi$ and $\lambda$ values) predictions and the $1 / H^{3}$ expected at low field is never observed, but we perfectly reproduce the experimental behavior by introducing our $\xi_{a b}(H)$ and $\lambda_{a b}(H)$ values in the numerical interpolation formulas calculated by $\mathrm{Brandt}^{28}$ with only one scaling factor due to the fact that the value of the number of pins is unknown.

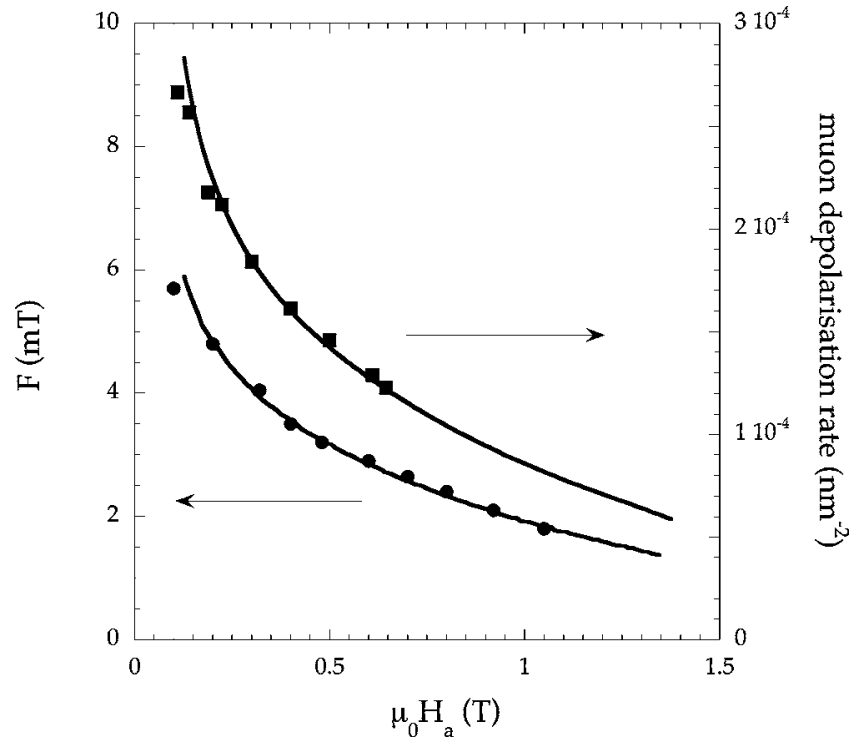

FIG. 4. Magnetic field dependence of the form factor in SANS experiments (circles) and of the muon spin relaxation rate (squares) compared to the calculated values (solid lines) with $\xi(H)$ and $\lambda(H)$ displayed in Figs. 1 and 2.

\section{Neutron scattering and muon depolarization experiments}

Having determined $\xi(H)$ and $\lambda(H)$ from our experiments, it is now possible to look for the consequences of those field dependences on other experimental quantities. First, in muon spin rotation experiments, the depolarization rate is proportional to $\sigma$ where $\sigma^{2}$ is the variance of the internal field and

$$
\sigma=0.07 \Phi_{0} / \lambda^{2} f(h)
$$

[where $f(0)=1$ and $f(h) \sim 0.45 \times(1-h)$ for $h>0.5$ (Ref. 25)]. Neglecting the field dependence of $\xi$ and hence assuming that $h \ll 1$, Angst et al. ${ }^{9}$ attributed the field dependence of $\sigma$ directly to $\lambda(H)$ which led to an overestimation of $\lambda_{a b}$ similar to the one discussed above. The influence of two gap superconductivity on the muon relaxation rate has also been discussed by Serventi et al. ${ }^{27}$ assuming that the contributions of the two bands could be added independently. Figure 4 displays the calculated field dependence of $0.07 \Phi_{0} / \lambda_{a b}^{2} f(h)$ deduced from our $\xi_{a b}(H)$ and $\lambda_{a b}(H)$ values without any additional free parameter [taking $f(h)$ from Ref. 26]. As shown, this function perfectly reproduces the experimental behavior. It is important to note that due to the field dependence of $\xi$, $h$ is never much smaller than 1 .

Second, as shown in Fig. 4, we can also very well reproduce the field dependence of the form factor in small angle neutron scattering experiments

$$
F=\frac{\sqrt{3}}{2} \frac{1}{4 \pi^{2}} \frac{\Phi_{0}}{\lambda^{2}} f^{\prime}(h)
$$

Instead of the exponential approximation taken in Ref. 4, we used the exact $f^{\prime}(h)$ function calculated by Brandt. ${ }^{26} \mathrm{We}$ again perfectly reproduce the field dependence of the experimental data. However, in this case, we had to introduce a scaling factor which might be due either to a different $\lambda_{a b}(0)$ 
value in our crystal (about 30\% smaller than theirs) and/or to some quantitative uncertainty in the determination of $F$ from SANS data.

\section{CONCLUSION}

In summary, we have shown that specific heat, magnetization, small angle neutron scattering, and muon spin relaxation measurements can be consistently described by assuming that the coherence length and the penetration depth are both field dependent in magnesium diboride due to the pro- gressive decrease of the contribution of the $\pi$-band. Superconductivity in this latter band is however possible up to $H_{c 2} \sim H_{c 2}^{\sigma}$ due to the coupling with the $\sigma$-band. To the contrary of most approaches, we have thus analyzed the experimental data by taking a continuous and smooth field dependence of $\xi$ and $\lambda$ all the way up from $H_{c 1}$ to $H_{c 2}$. This field dependence can be analyzed with the two-band GinzburgLandau model by assuming that the order parameters of the two bands are related together by a field dependent constant $\rho(H)\left(\psi_{\pi}=\rho \times \psi_{\sigma}\right)$ which is tending towards zero for $H_{a}$ $\rightarrow H_{c 2}$. By neglecting the field dependence of $\xi$, previous authors largely overestimated $\lambda .^{2,4,9}$
${ }^{1}$ L. Lyard et al., Phys. Rev. B 66, 180502(R) (2002); S. L. Bud'ko, V. G. Kogan, and P. C. Canfield, ibid. 64, 180506 (2001), M. Angst, R. Puzniak, A. Wisniewski, J. Jun, S. M. Kazakov, J. Karpinski, J. Roos, and H. Keller, Phys. Rev. Lett. 88, 167004 (2002), U. Welp et al., Phys. Rev. B 67, 012505 (2003).

${ }^{2}$ L. Lyard, P. Szabo, T. Klein, J. Marcus, C. Marcenat, K. H. Kim, B. W. Kang, H. S. Lee, and S. I. Lee, Phys. Rev. Lett. 92, 057001 (2004).

${ }^{3}$ P. Szabó et al., Phys. Rev. Lett. 87, 137005 (2001); P. Samuely et al., Physica C 385, 244 (2003).

${ }^{4}$ R. Cubitt, M. R. Eskildsen, C. D. Dewhurst, J. Jun, S. M. Kazarov, and J. Karpinski, Phys. Rev. Lett. 91, 047002 (2003).

${ }^{5}$ M. Eisterer, M. Zehetmayer, H. W. Weber, and J. Karpinski, Phys. Rev. B 72, 134525 (2005).

${ }^{6}$ L. Lyard, T. Klein, J. Marcus, R. Brusetti, C. Marcenat, M. Konczykowski, V. Mosser, K. H. Kim, B. W. Kang, H. S. Lee, and S. I. Lee, Phys. Rev. B 70, 180504(R) (2004).

${ }^{7}$ V. G. Kogan, Phys. Rev. B 66, 020509(R) (2002); V. G. Kogan and N. V. Zhelezina, ibid. 69, 132506 (2004).

${ }^{8}$ F. Bouquet, Y. Wang, L. Sheikin, T. Plackowski, A. Junod, S. Lee, and S. Tajima, Phys. Rev. Lett. 89, 257001 (2002).

${ }^{9}$ M. Angst, D. DiCastro, D. G. Eshchenko, R. Khasanov, S. Kohout, I. M. Savic, A. Shengelaya, S. L. Bud'ko, P. C. Canfield, J. Jun, J. Karpinski, S. M. Kazarov, R. A. Ribeiro, and H. Keller, Phys. Rev. B 70, 224513 (2004).

${ }^{10}$ M. Nohara, M. Isshiki, F. Sakai, and H. Takagi, J. Phys. Soc. Jpn. 68, 1078 (1999).

${ }^{11}$ T. Klein, C. Marcenat, F. Bouquet, A. Junod, S. Blanchard, and J. Marcus, Physica C 408-410, 731 (2004).

${ }^{12}$ D. A. Wright, J. P. Emerson, B. F. Woodfield, J. E. Gordon, R. A. Fisher, and N. E. Phillips, Phys. Rev. Lett. 82, 1550 (1999); K. Izawa, A. Shibata, Y. Matsuda, Y. Kato, H. Takeya, K. Hirata, C. J. van der Beek, and M. Konczykowski, Phys. Rev. Lett. 86, 1327 (2001).

${ }^{13}$ V. G. Kogan and N. V. Zhelezina, Phys. Rev. B 71, 134505
(2005).

${ }^{14}$ F. D. Callaghan, M. Laulajainen, C. V. Kaiser, and J. E. Sonier, Phys. Rev. Lett. 95, 197001 (2005).

${ }^{15}$ T. Yokoya et al., Science 294, 2518 (2001); J. G. Rodrigo and S. Vieira, Physica C 404C, 306 (2004).

${ }^{16}$ E. Boaknin, M. A. Tanatar, J. Paglione, D. Hawthorn, F. Ronning, R. W. Hill, M. Sutherland, L. Taillefer, J. Sonier, S. M. Hayden, and J. W. Brill, Phys. Rev. Lett. 90, 117003 (2003).

${ }^{17}$ A. V. Sologubenko, J. Jun, S. M. Kazakov, J. Karpinski, and H. R. Ott, Phys. Rev. B 66, 014504 (2002).

${ }^{18}$ M. R. Eskildsen, M. Kugler, S. Tanaka, J. Jun, S. M. Kazarov, J. Karpinski, and O. Fischer, Phys. Rev. Lett. 89, 187003 (2002).

${ }^{19}$ M. E. Zhitomirsky and V. H. Dao, Phys. Rev. B 69, 054508 (2004).

${ }^{20}$ Z. Hao and J. R. Clem, Phys. Rev. Lett. 67, 2371 (1991).

${ }^{21}$ E. H. Brandt, Phys. Rev. B 68, 054506 (2003).

${ }^{22}$ M. Zehetmayer, M. Eisterer, J. Jun, S. M. Kazakov, J. Karpinski, and H. W. Weber, Phys. Rev. B 70, 214516 (2004).

${ }^{23}$ A. A. Golubov, A. Brinkman, O. V. Dolgov, J. Kortus, and O. Jepsen, Phys. Rev. B 66, 054524 (2002).

${ }^{24}$ D. Pal, L. De Beer-Schmitt, T. Bera, R. Cubitt, C. D. Dewhurst, J. Jun, N. D. Zhigadlo, J. Karpinski, V. G. Kogan, and M. R. Eskildsen, Phys. Rev. B 73, 012513 (2006).

${ }^{25}$ E. H. Brandt, Phys. Rev. Lett. 66, 3213 (1991) and references therein.

${ }^{26}$ E. H. Brandt, Phys. Rev. Lett. 78, 2208 (1997).

${ }^{27}$ S. Serventi, G. Allodi, R. DeRenzi, G. Guidi, L. Romano, P. Manfrinetti, A. Palenzona, C. Niedermayer, A. Amato, and C. Baines, Phys. Rev. Lett. 93, 217003 (2004).

${ }^{28}$ E. H. Brandt, Phys. Rev. Lett. 57, 1347 (1986) ( $\alpha \neq 0$ case).

${ }^{29}$ G. Blatter, M. V. Feigel'man, V. B. Geshkenbein, A. I. Larkin, and V. M. Vinokur, Rev. Mod. Phys. 66, 1125 (1994).

${ }^{30}$ J. Gilchrist and M. Konczykowski, Physica C 212, 43 (1993); G. Pasquini, L. Civale, H. Lanza, and G. Nieva, Phys. Rev. B 59, 9627 (1999). 